\title{
SCANNING FORCE MICROSCOPY STUDIES OF IMPLANTED SILICON CRYSTALS
}

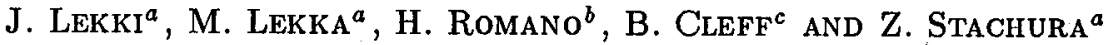 \\ ${ }^{a}$ Institute of Nuclear Physics, Radzikowskiego 152, 31-342 Kraków, Poland \\ ${ }^{b}$ Ecole Centrale Paris, Grande Voie des Vignes \\ 92295 Chatenay-Malabry Cedex, France \\ 'Institute of Nuclear Physics, Wilhelm-Klemm-Str. 9, 48-149 Münster, Germany
}

Scanning force microscope has been applied to investigate wear tracks produced during friction coefficient measurements of hard steel ball against 〈111〉 silicon crystals implanted with Ar ions. Such treatment causes the stable and significant decrease in friction, despite the total removal of implanted species from the wear track during friction. Scanning force microscope measurements of wear tracks topography supported the former hypothesis assuming the formation of post-implantation dense microcracks structure and subsequent propagation of this structure into the bulk. Such process assures small size of wear particles and a low friction coefficient value. Additionally the microfriction force measurement method was applied to determine the friction coefficient of $\mathrm{Si}_{3} \mathrm{~N}_{4}$ cantilever and a wear track in Si crystal.

PACS numbers: $62,20 .-\mathrm{x}$

\section{Introduction}

Numerous, well known and broadly reported advantages of ion implantation make this technique highly interesting both for industrial applications and for research. In the field of tribology ion implantation may cause changes in friction coefficient and microhardness or improve wear behaviour, increase adhesion of thin films to the substrate or improve corrosion resistivity. However, interpretation of results is often not easy because of large complexity of investigated physical system and many factors influencing tribological systems: morphological, physical and chemical. Therefore gathering a broad spectrum of data characterizing the investigated systems and the use of several complementary experimental techniques is highly desirable. In our former experiment [1], in order to simplify the investigated system, silicon single crystals were chosen. The obtained results, shortly discussed in the next paragraph, were clarified by the additional studies with the use of scanning force microscope (SFM). 


\section{Experimental}

Several samples were implanted in the mass separator facility of the Institute of Nuclear Physics in Cracow [2] with argon ions of energy $70 \mathrm{keV}$ up to the dose of $10^{17}$ ions $/ \mathrm{cm}^{2}$. Argon was chosen to avoid chemical effects associated with implanted ions. The implantation dose was high enough to ensure the total amorphization of the surface layer.

The implantation had the dramatic effect on the friction coefficient measured in vacuum with the use of a standard pin-on-disc setup (as a counterbody for silicon crystals a hard steel ball was used): a significant drop of friction coefficient from a value of roughly 1.0 for unimplanted samples, to 0.4 for Ar implanted (see Fig. 1).

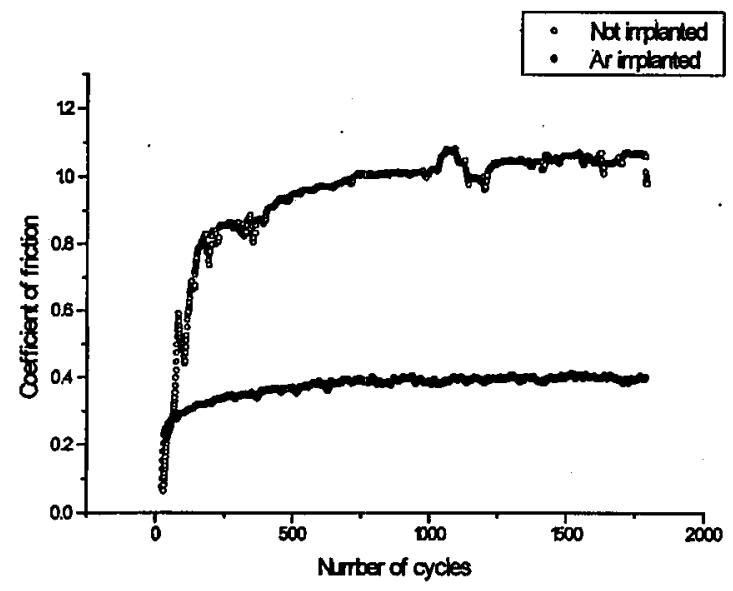

Fig. 1. Friction coefficient of not implanted (0) and implanted ( $\bullet$ ) Si crystals, measured in vacuum.

The effect was stable in time and the friction coefficient remained low even after a long time and complete removal of Ar from the track due to the wear. The possibility of Ar migration into the bulk during friction process was excluded after performing micro-RBS (Rutherford backscattering) measurements showing the depth profile of surface layer in the wear tracks region. This indicates that the decrease in friction coefficient was not simply due to the presence of implanted atoms. Additionally, the wear tracks of implanted samples measured with the conventional stylus profilometer had the surface much more smooth and regular than not implanted ones.

The decrease in friction coefficient was explained in the following way: damage introduced by high dose implantation leads to formation of microcracks defining future wear particles. As a consequence, subsequent removal of material during the wear process requires less force than in the case of unimplanted silicon, where the upper layer has the undistorted crystal structure. According to this simple model, the suggested explanation assumes propagation of this defect structure into the bulk during the wear process. This effect assures low friction even after significant wear. 


\section{Results of SFM studies}

In further study of ion implanted silicon we have applied our home made scanning force microscope [3]. The instrument uses the laser beam deflection technique and works in contact mode. Coarse approach is performed by an InchWorm stepping motor (Burleigh Instr.). Fine approach and scanning is performed using a commercial piezo tube sectioned into four quadrants (Staveley Inc.). As scanning tips microfabricated $\mathrm{Si}_{3} \mathrm{~N}_{4}$ cantilevers (Park Scientific Instr.) are used. Data acquisition and processing system uses a 486 personal computer equipped with two ADC/DAC cards (National Instr.). An overview of the construction is presented in Fig. 2.

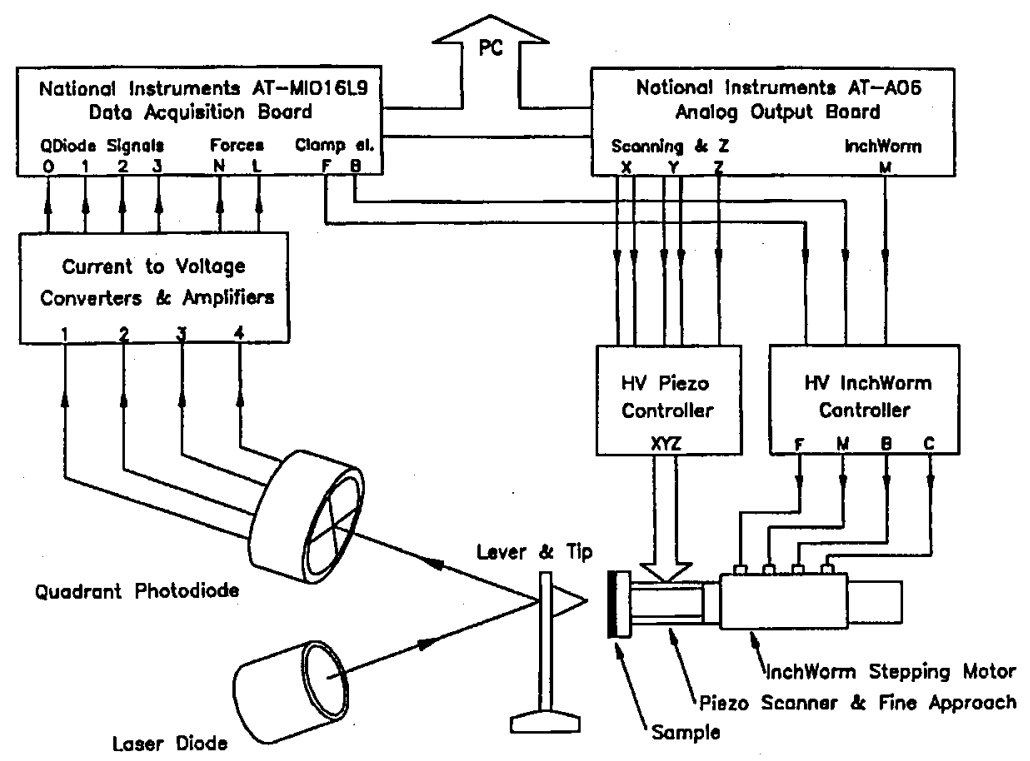

Fig. 2. Scanning force microscope of the Institute of Nuclear Physics in Cracow.

Wear tracks corresponding to not implanted and implanted samples after friction measurements performed in vacuum have been imaged using the SFM. All SFM measurements of wear tracks were done in air. The subsequent images represent normal force scans and thus contain information about surface morphology. In addition to images of the bottom area of tracks, Fig. 3 presents the wear track edge.

This picture gives a comparison between initial condition of silicon surface and its state after friction test. On smooth, untouched areas no significant differences between implanted and not implanted samples were found. This region of silicon crystal was smooth with some small particles attached to the surface. Only due to the presence of these particles the peak-to-peak value was in the range of 200-300 $\mathrm{nm}$. The average surface roughness was about $18 \mathrm{~nm}$, and the surface 


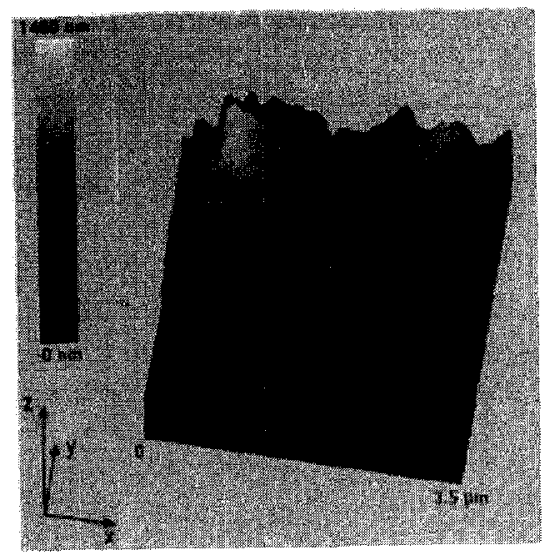

Fig. 3. Transition area between flat, unluuched Si surface and the wear track. (Coordinates: $x, y$ - distance, $z$ - height).
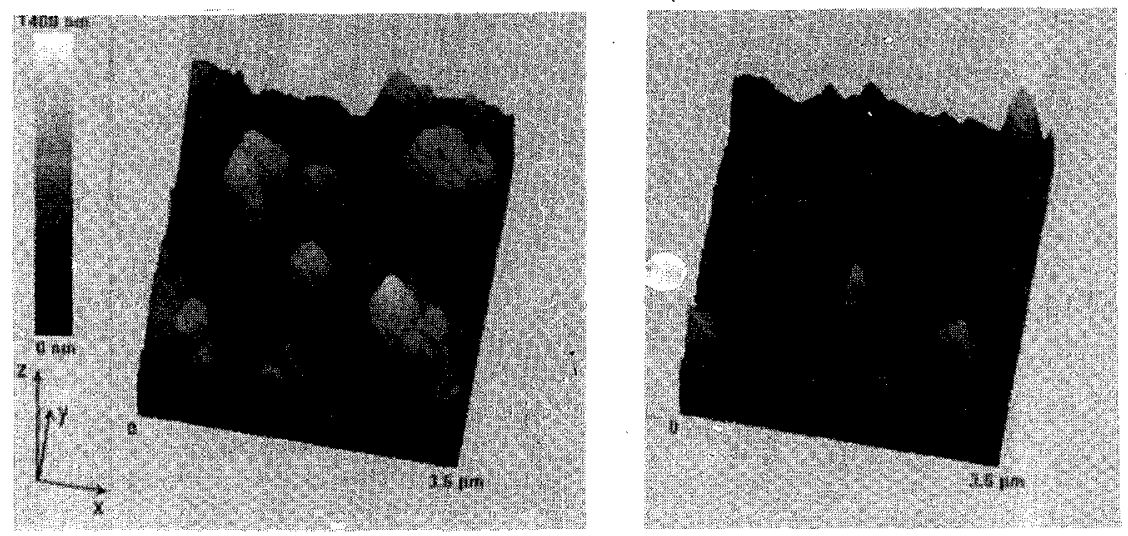

Fig. 4. Wear tracks topography for friction measurement performed in vacuum. Left - not implanted, right - implanted silicon sample. (Coordinates: $x, y$ - distance, $z$ - height).

root mean square $25 \mathrm{~nm}$. The definitions of these parameters as applied to SFM topographical images have been listed in [4].

Figure 4 and Table show the comparison between the topography of not implanted and implanted silicon wear tracks produced in vacuum. The difference in samples topography is very pronounced. Typical size of surface details are presented. It is clearly seen that in the case of implanted sample the size of particles present in the wear track is much smaller than for not implanted one. Therefore the cracks defining wear particles are much smaller than in the case of not irradiated sample. The microscopic images obtained with the SFM are therefore consistent with the explanation proposed in [1]. 
TABLE

Main topographical parameters of wear tracks of samples measured using pin-on-disc setup in vacuum. Values in parenthesis correspond to mean value errors.

\begin{tabular}{c|c|c|c}
\hline \hline Friction in vacuum & Peak-to-peak & Average roughness & Root mean square \\
\hline Not implanted & $1610(40)$ & $204(16)$ & $257(17)$ \\
Ar implanted & $1140(100)$ & $95(4)$ & $126(6)$
\end{tabular}

Finally, friction coefficient for implanted and not implanted samples was estimated. Of course, friction studied by SFM has nothing to do with friction measured using the pin-on-disc setup. SFM measures frictional forces between the surface and silicon nitride tip in totally different conditions. The following piactical example illustrates the method of friction coefficient determination.

Following Ref. [5], the lateral spring constant of the cantilevers used in experiment has been estimated to $85 \mathrm{~N} / \mathrm{m}$. In angular representation the lateral force and cantilever torsion angle follow the dependence $\phi=3.9 \times 10^{3} F_{\text {lat }}$. The friction force may be extracted from the lateral force image and separated from topographical component by subtraction of the data sets corresponding to opposite directions of scanning (left-right and right-left) [6]. Both images are collected during single measurements. The topography of a sample, in the sense of $\mathrm{d} S / \mathrm{d} x$, where $S(x, y)$ is the shape of the sample surface and $x$ is the direction of scanning, may be easily obtained from normal force scan. An example, corresponding to scans presented later in Fig. 6 is shown below (Fig. 5).

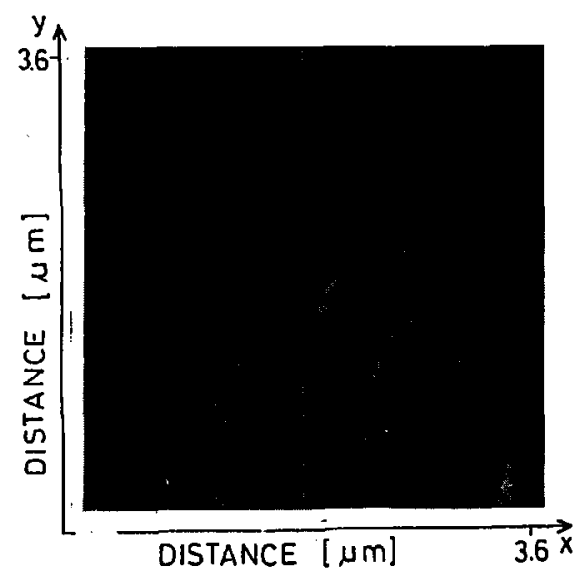

Fig. 5. Wear track surface derivative with respect to the direction of scanning. Other views of this image are presented in subsequent figures. (Coordinates: $x, y-$ distance).

Unfortunately, the piezo hysteresis present in left-right and right-left scan images causes that they are shifted relative to each other. Therefore subtracted image would be distorted and not suitable for calculations (see the left image of 
Fig. 8). Applying the method presented in [7], two images may be "synchronized" using simple image processing. The idea is to introduce a shift of one of the images along direction of scanning. The shift should cancel the effect of piezo hysteresis, at least in the central region of subtracted image, which is shown in the right part of Fig. 8. Only then the friction may be reasonably estimated.
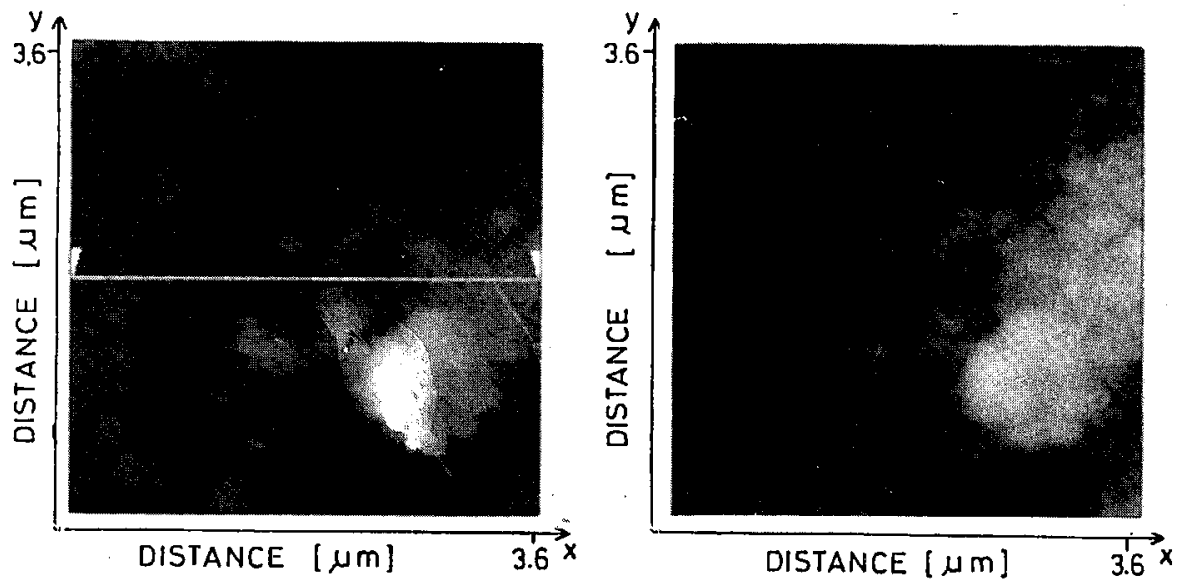

Fig. 6. Left - topography image, normal force. White line shows the position of cross-section (see next figure). Right - scanned simultaneously lateral force image. For this sample the presence of topography in lateral force image is very significant. (Coordinates: $x, y$-distance).
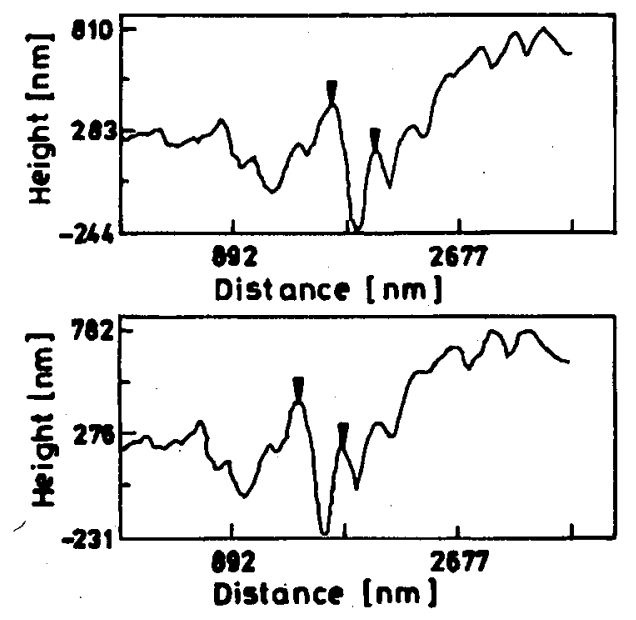

Fig. 7. Cross-sections of complementary images (see left part of the previous figure). Top image - scanning from left (minimal voltage) to right. Bottom - opposite. 

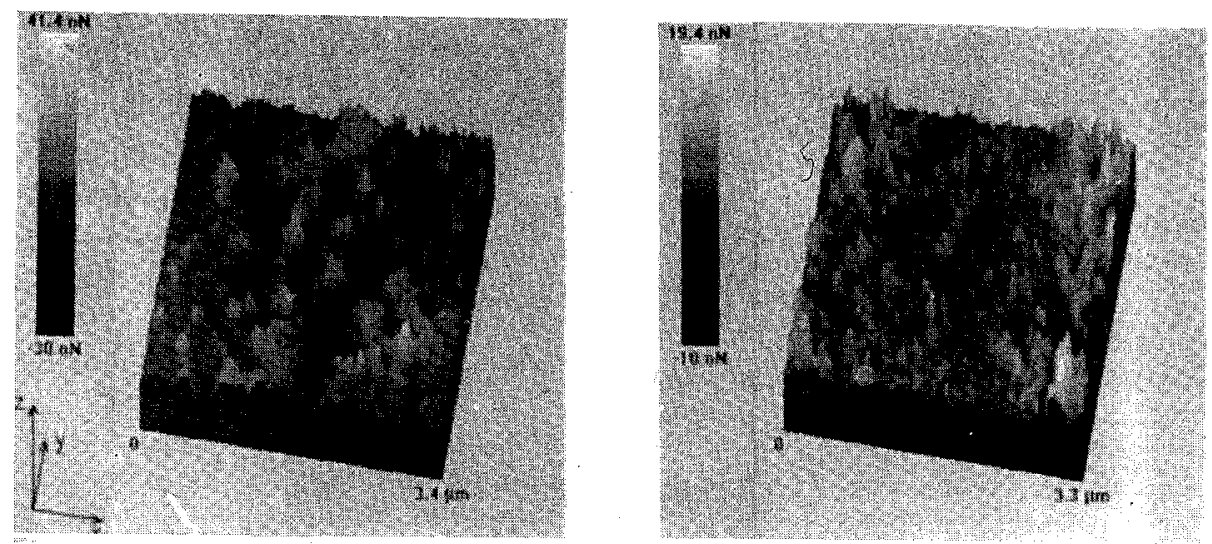

Fig. 8. Left - result of image subtraction without any processing. Piezo scanner imperfections are responsible for resulting artifacts. Right - subtraction after synchronization of complementary images. (Coordinates: $x, y$ - distance, $z$ - friction force).

The image processing algorithm used above is quite straightforward. First, the rough estimation of image shift is done, using horizontal cross-sections of the complementary images. An example is shown in Fig. 6 and Fig. 7.

The shift can be estimated to about $240 \mathrm{~nm}$ (please note the positions of markers). This distance corresponds to 17 image pixels. For this measurement one scanning step (and therefore also one image pixel) was equal to $14 \mathrm{~nm}$. Then, original lateral force images are synchronized by shifting of one of it by 17 pixels. After subtraction the uniform central region is a measure of pure friction force multiplied by a factor of 2 with the removed topographical component (right part of Fig. 8).

The friction force measured in the central area region of the right image, after dividing by 2 , has the mean value of $4 \mathrm{nN}$. For the loading force in the range of tenths of $\mathrm{nN}$ many papers $[8,9,6,10]$ prove the linear dependence of the SFM lateral force value and the loading force, therefore simple friction equation $F_{\mathrm{F}}=$ const $+\mu F_{\mathrm{L}}$ is justified. The average loading force was in this measurement about $20 \mathrm{nN}$. Therefore for a $\mathrm{Si}_{3} \mathrm{~N}_{4}$ cantilever against a wear track of $\mathrm{Ar}$ implanted $\mathrm{Si}$ crystal the lateral force divided by loading force gives a friction coefficient value of 0.2 . This may be compared with the other $\mathrm{Si}_{3} \mathrm{~N}_{4}$ microfriction coefficient values reported for:

- mica: $\mu=0.09$ [9],

- diamond film on a Si substrate: $\mu=0.18$ [6],

- gold film on mica: $\mu=0.16-0.21$ [6],

- ITO (indium-tin-oxide) film on glass: $\mu=0.11$ [6].

However, the reproducibility of our measurements is rather poor $( \pm 30 \%)$, most probably due to the surface contamination (all measurements were done in ambient air). However no detailed statistical analysis has been performed. 


\section{Conclusions}

Scanning force microscope is an excellent tool for microtribology investigations. It brings three-dimensional detailed information on surface topography in the form which is very suitable to subsequent data processing. As it was shown the SFM images may be very helpful in interpreting tribological experiments. Additionally, SFM lateral force images contain information on friction forces which can be extracted using relatively simple techniques. Finally, one should mention about another possibility of great importance for friction studies: the measuring of adhesion forces. This topic, however not covered by the present article, will be a subject of our studies in the nearest future.

\section{Acknowledgments}

SFM construction was performed in a close collaboration with the Institute of Nuclear Physics (IKP) of Münster University. The project was financed by the Committee for Scientific Research grant No. 2P30204505. Personal costs of foreign visits were financed by the Scientific and Technical Collaboration between Germany and Poland, project No. X-082.2.

\section{References}

[1] J. Lekki, Z. Stachura, N. Preikschas, B. Cleff, M. Cholewa, G. Legge, J. Mater. Res. 9, 91 (1994).

[2] B. Rajchel, M. Drwięga, E. Lipińska, M. Wierba, Nucl. Instrum. Methods Phys. Res. B 89, 342 (1994).

[3] J. Lekki, U. Voß, M. Sowa, B. Cleff, Z. Stachura, INP Report 1690/AP, 1995.

[4] J.F. Joergensen, K. Carneiro, L.L. Madsen, Nanotechnology 4, 152 (1993).

[5] J.M. Neumeister, W.A. Ducker, Rev. Sci. Instrum. 65, 2527 (1994).

[6] S. Grafström, M. Neitzert, T. Hage, J. Ackermann, R. Neumann, O. Probst, M. Wörtge, Nanotechnology 4, 143 (1993).

[7] M. Labardi, M. Allegrini, M. Salerno, C. Fredriani, C. Ascoli, Appl. Phys. A 59, 3 (1994).

[8] A.J. Boef, Rev. Sci. Instrum. 62, 88 (1991).

[9] R.J. Warmack, X.-Y. Zheng, T. Thundat, D.P. Allison, Rev. Sci. Instrum. 65, 394 (1994).

[10] M. Sasaki, K. Hane, S. Okuma, Y. Bessho, Rev. Sci. Instrum. 65, 1930 (1994). 